\title{
La Formación de la Comunidad de Inteligencia Española: Un proceso en marcha
}

\author{
Francisco Galvache Valero
}

Arbor CLXXX, 709 (Enero 2005), 183-205 pp.

El fin de la bipolaridad dio lugar a la aparición de nuevos riesgos y amenazas, y en un escenario en el que la globalización habia potenciado la interdependencia en los espacios definidos por el binomio seguridaddefensa, lo que imponía la necesidad de cooperación entre aliados, para resolver crisis (que crecían en número) y afrontar amenazas de carácter cada vez más transnacional. La invasión de Kwait, preludio de la guerra de Irak, la larga crisis de los Balcanes, y el casi sempiterno conflicto de Oriente Medio, mostraron un potencial desestabilizador no previsible antes de la caída del muro de Berlín.

Estos cambios, unidos a los inducidos por la revolución de la información y de las comunicaciones, aconsejaron la reforma de los sistemas de inteligencia, para adaptarlos a la nueva y cambiante situación y a la necesidad de la cooperación, para dar respuestas globales a amenazas que eran globales. Tras el 11 de febrero, los fallos de coordinación que se advierten en la Comunidad de Inteligencia de Estados Unidos, hacen que la mayoría de los gobiernos impriman un nuevo ritmo a los procesos de reforma de sus respectivos sistemas, poniendo especial énfasis en las arquitecturas de coordinación que vertebran las comunidades de inteligencia.

Por lo que respecta a España, el gran problema de la coordinación se aborda, por primera vez, durante la elaboración y posterior promulgación de la Ley 11/2002 de 6 de mayo, Reguladora del CNI, por la que se crea la Comisión Delegada del Gobierno para Asuntos de Inteligencia (CDGAI) la figura de la Autoridad Nacional de Inteligencia y Contrainteligencia y el Centro Criptológico Nacional. Hasta entonces, no se podía hablar, con rigor, de la existencia en nuestro país de una Comunidad de 
Inteligencia. Hoy, bajo la tutela de la CDGAI, su proceso de formación continua en marcha.

\section{Introducción}

El término inteligencia es, ciertamente, polisémico. En un sentido antropológico es sinónimo de entendimiento, capacidad específica del ser humano que le permite conocer su propia realidad y la del mundo que le circunda, y hacerse cargo de una y de otra. Una capacidad que, unida esencialmente a la memoria y a la voluntad, le dota de sentido biográfico y le constituye en protagonista de su propia vida: en autor y actor de su propio drama vital. La inteligencia, pues, produce conocimiento; la memoria acumula tal experiencia; y la voluntad -donde reside la capacidad de querer- decide desencadenar la acción y perseverar en la realización de lo decidido.

Todo lo que deviene del hombre (la familia, la sociedad, el Estado...) guarda su impronta y, por tanto, mantiene con él lógicas analogías. Pues bien, en el ámbito del Estado, ocurre algo parecido en el curso de las actividades que debe desarrollar el poder ejecutivo para garantizar la gobernabilidad en todo lo concerniente a los objetivos estratégicos que haya de plantearse. Para ello necesitará de la inteligencia ${ }^{1}$, del conocimiento específico y adecuado a cada caso y, por tanto, de los órganos capaces de elaborar y aportar dicho conocimiento para, a partir de ahí, poder decidir las políticas que deban orientar y modular las acciones de los diferentes organismos de la administración del Estado en los ámbitos de sus respectivas competencias, dotarles de las capacidades necesarias y, por último, establecer los principios y mecanismos que permitan e impulsen la acción de conjunto de aquellos actores que deban dirigir sus esfuerzos al logro de objetivos compartidos. En resumen: en una primera acepción, el término inteligencia es sinónimo de conocimiento; pero, en tanto que entidad y proceso capaz de producirlo, también supone organización e implica actividad. Y así cabe hablar, con toda propiedad, en nuestro caso, de servicios y de actividades de inteligencia.

\footnotetext{
1 En el diccionario de la RAE: a.2: «Conocimiento, comprensión, acto de entender».
} 
En la organización y funcionamiento del Estado, al Gobierno le atañe -le corresponde- promover el bienestar, los derechos y las libertades de los ciudadanos y, de manera primordial, garantizar su seguridad y la defensa de la Nación como tal, y la de sus intereses generales. En consecuencia, su acción debe orientarse al logro de un amplio abanico de objetivos relacionados con tales valores; objetivos entre los que se cuentan algunos de importancia decisiva, es decir, los objetivos estratégicos ${ }^{2}$ : objetivos que, por su trascendencia, exigen desarrollar procesos de decisión de singular delicadeza que han de estar alimentados por el conocimiento pertinente, relevante, oportuno y discreto, por la información suficientemente contrastada y debidamente evaluada, es decir: por la Inteligencia Estratégica Nacional entendida como:

Conocimiento necesario para la toma de decisiones óptimas por parte del gobierno de la Nación, y en orden a alcanzar los objetivos que considera de importancia decisiva (estratégicos) para la seguridad y la defensa de los intereses nacionales en cada momento.

La elaboración, pues, de esta forma peculiar de conocimiento que denominamos inteligencia, requiere, como es natural, la existencia de

Una organización compleja, de nivel nacional, jerarquizada, especializada, al servicio del Estado y dotada, de la arquitectura y de los medios humanos y materiales adecuados a tal fin; $y$, por tanto, capaz de obtener información, analizarla, evaluarla y proporcionar la inteligencia necesaria para la toma de las decisiones de gobierno en los campos de la seguridad y de la defensa.

En resumen: una institución del Estado, dependiente del Gobierno de la Nación y capaz de desarrollar el proceso completo de lo que conocemos como Ciclo de Inteligencia. En síntesis, en esto, y no en otra cosa, consiste la entidad y función de un servicio nacional de inteligencia.

\section{El marco de actuación de los servicios de inteligencia}

Desde el punto de vista funcional, la inteligencia -que ya hemos visto que es conocimiento y es organización- también es actividad. Actividad que, que, como bien dice Esteban Navarro, «puede (y debe) enten-

\footnotetext{
2 El diccionario de la RAE define el término estratégico, en su segunda acepción, como término que adjetiva a «un lugar, posición, actitud, etc.. de importancia decisiva para el desarrollo de algo»; y lo hace, lógicamente, en el marco del concepto de estrategia, en tanto que "conjunto de reglas que aseguran una decisión óptima en cada momento».
} 
derse como un típico proceso de gestión de conocimiento» ${ }^{3}$ que, como ya he apuntado, se desarrolla en los espacios definidos por el binomio $S e g u$ ridad-Defensa.

Y llegados a este punto, aunque ello es bien sabido, quizá convenga recordar que la seguridad es una aspiración y también un sentimiento inherente a la condición humana. Es una aspiración que se ha de satisfacer de manera suficiente, porque de ello depende, en gran medida, el éxito o el fracaso de los proyectos vitales de las personas y de las sociedades. Por otra parte, la seguridad ideal, el sentimiento pleno -individual y colectivo- de hallarse a cubierto de cualquier agresión o daño, solo derivaría de una situación en la que no tuviese cabida.

"amenaza alguna a la soberanía ni a la integridad del territorio y sus habitantes; una situación en la que no existe atentado alguno contra el ejercicio normal de la autoridad ni contra el funcionamiento adecuado de las instituciones; y una situación en que tanto las actividades públicas como las privadas, pueden llevarse a cabo sin obstáculos que se opongan al logro de los más altos niveles de paz, libertad, prosperidad cultural, cívica, moral y económica» (Dirección General de Política de Defensa, 1993)

Esta descripción coincide sustancialmente con la que luego dibujaría el Concepto Estratégico de la Alianza Atlántica, aprobado en Washington en abril de 1999, al declarar la importancia, en materia de seguridad, de los factores políticos, económicos, sociales y medioambientales sujetos a riesgos, y la de aquellos otros, de naturaleza diversa, que contempla la defensa, tales como los que, desde el exterior o desde el interior, afectan directa o indirectamente a la soberanía nacional y a la integridad territorial de los estados, relacionados con frecuencia con los riesgos derivados del terrorismo nacional e internacional, de las actividades delictivas del crimen organizado, de los posibles colapsos en el flujo de los recursos vitales o de los grandes movimientos incontrolados de población -especialmente los generados por los conflictos armados- en razón de los graves problemas de seguridad y de estabilidad que plantean ${ }^{4}$ en un mundo de acusada complejidad y cada vez más interdependiente.

3 M.G. Esteban Nabarro y D. Navarro Bonilla: «Gestión del conocimiento y Servicios de Inteligencia: La dimensión estratégica de la Información". El profesional de la Información, vol. 12, $\mathrm{n}^{\circ} 4,2003$, pp. 269-291.

${ }^{4}$ Cfr. Concepto Estratégico de la Alianza, 1999, "Guía completa de la Cumbre de Washington» párrafo 25 , p. 55 . 
La Formación de la Comunidad de Inteligencia Española:...

El escenario general

Ciertamente, el proceso de globalización lleva muchos siglos desarrollándose. Y lo ha hecho, a lo largo del tiempo, de forma cada vez más acelerada, a impulsos del progreso del conocimiento en los campos de las ciencias y de las tecnologías al servicio del comercio, de la industria y de las comunicaciones. Pero como dijera Friedman ${ }^{5}$, hoy sus efectos llegan más lejos, más rápidamente, a menor costo y con una capacidad de penetración jamás alcanzada en la historia. La interdependencia, pues, es cada vez más intensa, más insoslayable, a medida que aumenta la espesura de las redes de interconexión en que se sustenta. De manera que, hoy, la autarquía aislacionista es imposible, y ceder a su tentación resultaría suicida.

En la actualidad, cualquier suceso acaecido en un subsistema resuena con mucha más fuerza, produce mayores efectos en el sistema global del que forma parte, y lo hace con mucha más rapidez que en cualquiera otra época del pasado. Como recuerda Nye, el virus de la viruela tardó casi tres milenios en alcanzar a todos los continentes, y la plaga del SIDA en menos de tres décadas se ha extendido por todo el planeta. Pues bien, en el año 2000, un virus -esta vez informático- a través de la red (verdadero sistema arterial del mundo globalizado) perturbó y causó daños en las comunicaciones, el comercio y los diferentes sectores productivos de todo el mundo en tan sólo tres días. «Tres milenios, tres décadas, tres días (concluye Nye): ésta es la medida en que aumenta la velocidad de la globalización» ${ }^{6}$.

Algo parecido ocurre con las noticias y con las ideas. Pero su comprensión y su asimilación o rechazo dependen, en gran medida, de las culturas que han de interpretarlas a la luz de los valores que a cada una le son propios. Y las culturas, como es bien sabido, evolucionan a ritmos muy diferentes de aquellos que rigen en los procesos de desarrollo de las ciencias aplicadas y de las nuevas tecnologías de la información. De manera que, a comienzos del siglo XXI se puede constatar, con claridad, que el proceso de la globalización no discurre y avanza únicamente impulsado por el vector económico, sino también a través de la formación y difusión de comunidades de ideas y de valores que informan y orientan los campos de la política, de la ciencia y de la cultura, y que van favorecien-

5 T. FRIEDMAN, The Lexus and the Olive Tree: Understanding Globalization, Farrar, Nueva York, 1999, Straus and Giroux, pp. 7-8.

6 J. NyE (2003) La paradoja el poder americano, Madrid, Taurus, 2003, p. 128. 
do la aparición y consolidación de intereses y objetivos comunes que alimentan esperanzadores procesos de convergencia. Y es que, como señala Beck, la globalización es un fenómeno poliédrico; y tratar de contemplarlo a través de una sola de sus facetas, por relevante que esta sea -y la económica lo es, sin duda- lo dejaría reducido a mero globalismo ${ }^{7}$.

En cualquier caso, es bien cierto que crece, día a día, el número de los países que perciben su destino y bienestar conectados a valores e intereses compartidos; y también lo es que va prosperando, a ritmo acelerado, en los gobiernos y pueblos de muy diferentes mundos y tradiciones, la convicción de que su promoción y su defensa exige la construcción de un nuevo orden mundial que, trascendiendo el realismo tradicional que desde Westfalia vino regulando las relaciones internacionales hasta la caída del muro de Berlín, se asiente en un espíritu abierto a la cooperación multilateral, a políticas de seguridad compartida y de defensa colectiva, e incluso a procesos de convergencia entre naciones-estado hacia nuevas estructuras políticas supranacionales que les permitan desarrollar y mantener políticas comunes, no sólo en el ámbito de la economía o en los ya aludidos campos de la seguridad y de la defensa, sino también en el de la justicia y aún en el de la política exterior. De aperturas de tal índole es un buen ejemplo el camino que vienen cubriendo las naciones europeas: un camino largo, zigzagueante e incluso azaroso; plagado de tensiones y desencuentros, pero que, a pesar de todo, avanza y gana en consistencia, suscita y consuma nuevas adhesiones e incluso asume el trascendental reto de construir, en común, un marco constitucional capaz de ser garantía de solidaridad, de justicia, de paz y de progreso.

Pero aceptando todo lo anterior, no es posible ignorar que, frente a estos fenómenos de convergencia, a partir de mediados del pasado siglo, vienen paradójicamente resurgiendo, en comunidades y pueblos, exacerbadas y aún sangrientas reacciones del romanticismo particularista del diecinueve, en sectores que pretenden actuar en defensa de una más o menos melancólica identidad histórica, racial o religiosa supuestamente amenazada; o, más recientemente, de corrientes ideológicas, grupos y organizaciones de orígenes frecuentemente periclitados, que configuran hoy un frente que vaticina sólo males del proceso de globalización en marcha; y desde el cual, algunos de sus integrantes no dudan, en ocasio-

7 Cfr. Ulrich BecK ¿Qué es la globalización? Paidos, Barcelona, 1998. Para Beck, la globalización es un proceso dirigido hacia una sociedad mundial, mientras que el globalismo sería un mero intento de reducir el fenómeno a tan sólo una de sus dimensiones: la económica. 
nes, traspasar el umbral de la acción política y de la legítima protesta, recurriendo a la violencia, incluida la de signo terrorista.

La vida del hombre, de las sociedades y de los pueblos ha sido siempre una realidad verdaderamente complicada: una maraña casi inextricable en la que la libre voluntad de los actores ha de enfrentarse constantemente con lo contingente regido por el principio de indeterminación. Una realidad en la que las capacidades definen posibilidades cuya realización se ve siempre favorecida o dificultada por funciones multivariables difíciles de prever, de detectar, de definir, y de evaluar, ajustadamente, en peso, desarrollo y consecuencias.

$\mathrm{Y}$ es que las coordenadas espaciotemporales entre las que discurre la contemporaneidad, hoy definen espacios y horizontes mucho más amplios y lejanos que en el pasado, en los que los individuos y las organizaciones sociales (más numerosas y diversas que nunca) interactúan a través de las redes que hoy tejen las nuevas tecnologías aplicadas a la información y a las comunicaciones. Redes que introducen, en las estructuras y en las dinámicas sociales, un grado tal de complejidad que hace más inabarcable y más interdependiente que nunca la realidad vital de nuestro tiempo.

Nuestra sociedad global, como dice A. Llano, es una sociedad en la que todo influye en todo; $y$, en consecuencia -se puede añadir-nadie debería pensarse al margen de lo que quizá ocurre a miles de kilómetros de distancia. Y menos aún si lo sucedido se halla en relación con riesgos, amenazas y agresiones que comprometen la paz y el bienestar de la humanidad, las libertades y los derechos humanos. En consecuencia, la seguridad y la defensa, salvaguardas de la paz y de la libertad, son asuntos que nos atañen a todos, que son responsabilidad de todos; y que, desde luego, lo son también de los servicios de inteligencia.

La caída del muro de Berlín tuvo resonancias considerables por cuanto determinó un acelerado proceso de cambio de las variables geopolíticas definidas en el marco de la bipolaridad, y, hasta entonces, actuantes en el juego de equilibrios logrados a través de las políticas de alianza y de disuasión diseñadas por las dos grandes potencias enfrentadas. Y, como era de esperar, tal proceso ganó velocidad y alcance tras la desintegración de la antigua Unión Soviética, y dio paso a una nueva situación impregnada de fuerte dinamismo, y que ofrece, en el tiempo, una imagen cambiante, desde luego, muy diferente de la del pasado, y más diferente aún de la que se apresuraron a forjar los fieles del optimismo impenitente.

Ciertamente, el ominoso enfrentamiento bipolar que amenazaba con la mutua destrucción, se había desvanecido; pero no ocurrió otro tanto 
con los riesgos, las amenazas ni con los conflictos abiertos que, por el contrario, se han diseminado, diversificado, y cuyo número no ha hecho sino crecer. El multilateralismo ha hecho acto de presencia, pero el escenario mundial hoy soporta la poderosa presencia de una superpotencia he gemónica en los planos político, militar y económico; el peso del grupo de las grandes potencias desarrolladas; el de un amplio abanico de países en vías de desarrollo (no a cubierto, en muchos casos, de riesgos ciertos de inestabilidad) y la nada despreciable rémora de un no escaso número de estados fallidos, incapaces de cumplir con el mínimo de las responsabilidades primordiales que justifican la razón de ser del Estado; y a estos hay que añadir aún otros -que se ha dado en llamar criminales- refractarios a la libertad, cuyas elites gobernantes se sostienen en el poder gracias a la represión y a la negación de los derechos humanos y cuya mera existencia proyecta amenazas de evidente gravedad para la estabilidad y para la paz de amplias regiones del mundo.

\section{Los riesgos y las amenazas}

Como se ve, pues, nuestra coyuntura está caracterizada por una notable disparidad de fuerzas y valores que alimenta la proliferación y diversificación de las áreas de conflicto, en las que crece el riesgo de que actores de tal naturaleza, conscientes de su situación de inferioridad ante sus adversarios, opten por el empleo de modelos de guerra sin restricciones: modelos de guerra que no reparan en medios ni discriminan objetivos. Modelos que les permiten, apoyados por aliados a los que haré referencia, y en las posibilidades que ofrece la globalización económica, de la información, de las comunicaciones y de las nuevas tecnologías militares de relativamente fácil acceso, albergar la esperanza de imponer su voluntad a enemigos regionales incluso más fuertes que ellos, y de, llegado el caso, exhibir su capacidad de disuasión y aún de respuesta ante las grandes potencias democráticas, en un intento de perpetuar su tiranía o de alcanzar sus objetivos más o menos utópicos.

Estos modelos de guerra sin restricciones, como es sabido, se basan en el empleo de procedimientos y formas de acción capaces de equilibrar la desproporción de fuerzas existente entre los contendientes; y pasan, por tanto, por el empleo de medios tales como: la amenaza y voluntad de uso, llegado el caso, de las armas de destrucción masiva, y/o del terrorismo directamente practicado o coordinado, mediante alianzas más o menos coyunturales, con las organizaciones terroristas que ya lo vienen practi- 
cando a escala internacional en pos de sus particulares designios. Alianzas que también se extienden al mundo de la criminalidad organizada (verdadero azote de los estados de derecho) dedicadas a tan lucrativas formas delictivas como el tráfico de estupefacientes, de armas cada vez más sofisticadas, de seres humanos, del fraude financiero a gran escala y de la corrupción política y económica a través de la extorsión y de la compra de voluntades.

Quizá la sociedad global de nuestro tiempo aún no sea capaz de valorar, con justeza, el potencial desestabilizador de este último fenómeno, en el plano doméstico y en el de las relaciones internacionales directamente relacionadas con la seguridad y con la defensa. Afortunadamente, otra cosa bien distinta ocurre con cuanto se refiere a los conflictos regionales que siembran la muerte, provocan éxodos masivos en las poblaciones afectadas, y cercenan sus posibilidades de progreso mientras las privan de acceso al flujo de los recursos vitales más indispensables, con las dramáticas secuelas del desarraigo, del hambre y de la enfermedad. Ciertamente, la gravedad e importancia de este trágico factor de inestabilidad ha ido calando con fuerza en cada vez más amplios sectores de las sociedades desarrolladas, haciéndoles ver, con nitidez, que nada de todo esto les puede ser ajeno.

Pero hay que reconocer que ha sido el fenómeno del terrorismo internacional, el que ha coadyuvado, de forma quizá determinante, a que las sociedades hayan tomado general conciencia de que se encuentran ante una amenaza global que es compartida (sufrida) por todos y cada uno de los países del mundo, porque no discrimina ni entre pueblos ni personas, porque su acción destructiva traspasa las fronteras o brota del mismo seno de las comunidades contra las que luego atenta, y porque, empapado de utopía, amenaza al mundo entero con el anuncio de un sombrío futuro de zozobra y de muerte del que no se vislumbra un fin cercano.

De la magnitud del fenómeno y de su carga letal, la comunidad internacional tiene ya una larga y dura experiencia de pasado. Para el presente, basten, como muestra, las mortales acciones que el terrorismo islamista -de cuyas organizaciones la red Al-Qaeda es el más trágico y más acabado ejemplo- proyecta y ejecuta, no solo contra las sociedades libres de Occidente que, tras una larga e inconclusa serie de atentados, hubieron de sufrir el horror del 11 de septiembre en Nueva York y Washington o la espantosa matanza del 11 de marzo en Madrid, sino también contra sus hermanos desviados; ya sea en Irak, Marruecos, Turquía, Arabia Saudita, Egipto, Afganistán, Pakistán, Indonesia o sobre regiones aún más aparentemente alejadas de la Base (al-Qaeda). 
Todo este cúmulo de circunstancias justifica plenamente la necesidad sentida en el concierto de las naciones libres de construir un nuevo orden mundial que, con el garante respaldo de Naciones Unidas y en términos acordes con la actual multilateralidad centrada en Estados Unidos, en las potencias democráticas y en aquellas otras que, sincera y decididamente, aspiran a serlo, haga posible instaurar una verdadera legalidad capaz de regular las relaciones en el seno de la comunidad internacional según los principios del realismo cooperativo, desde el cual quepa desarrollar políticas que, de forma equitativa y eficaz, afronten estos problemas en toda su complejidad, favorezcan la cooperación y la coordinación de esfuerzos y pongan, especial énfasis en la solidaridad real con los países del segundo y tercer mundo -en donde radican buena parte de las causas del fenómeno- y sean garantes de la libertad, de la seguridad y de la justicia, en tanto que fundamentos de la estabilidad, de la paz y del progreso de los pueblos.

\section{Hacia la reforma y la cooperación}

Tan importantes retos necesariamente habían de tener también importantes resonancias en las funciones y estructuras de los servicios de inteligencia quienes, de hecho, a partir del término de la guerra fría, ya habían iniciado procesos de adaptación a la situación geopolítica emergente, y a las nuevas orientaciones conceptuales que se fueron desarrollando en relación con la seguridad y con la defensa, urgidas por la aparición de los nuevos riesgos y amenazas, que, como ya hemos visto, son cada vez más numerosos, más diversos, difíciles de anticipar en no pocas ocasiones, y para cuya prevención, detección, neutralización y, en su caso, gestión, se comenzaba a ver, la inexcusable necesidad del esfuerzo concertado de las agencias. En definitiva, la fuerza de los hechos fue imponiendo y propiciando, progresivamente, una clara toma de conciencia acerca de la necesidad de dar respuestas globales a fenómenos que eran y que son globales.

Esta cuestión pasó, pues, a constituirse en nutriente principal de los debates en los foros y clubes de inteligencia, y aun en el seno de los propios servicios participantes en ellos. Se percibía cada vez con mayor claridad la conveniencia de incrementar la cooperación a través del intercambio de la información y de la inteligencia; Pero la desaparición de la gran amenaza común a los países alineados en cada uno de los antiguos grandes bloques, y la dificultad de valorar el alcance global de amenazas 
hasta entonces bastante localizadas y, en general, afrontadas por cada uno de ellos en sus respectivas áreas de influencia, o, en todo caso, por los países directamente afectados, ralentizaba el avance de los procesos de adaptación y reforma de los sistemas nacionales de inteligencia que hicieran posible la cooperación coordinada en el plano internacional; máxime cuando, a niveles nacionales, tal capacidad presentaba $-\mathrm{y}$ presenta aún- notables deficiencias.

Pero la realidad es terca y acabó imponiéndose, con toda su crudeza, tras los sucesos acaecidos el 11 de septiembre de 2001. En esa luctuosa fecha, se confirmó lo que ya anticipara Henry Kiesinger tiempo atrás: en el futuro previsible, ni siquiera la superpotencia hegemónica, Estados Unidos, podrá afrontar, en solitario, amenazas que, por su naturaleza y alcance, resulta imposible reducir al ámbito de lo doméstico porque lo trascienden y ponen en riesgo la paz y la estabilidad incluso mundiales.

En resumen, las funciones genéricas de los servicios de inteligencia se han visto obligadas a proyectarse sobre un mayor número de escenarios y sobre espacios que traspasan los límites que establecen las fronteras nacionales; y la constatada interdependencia, fruto de la globalización de los riesgos y de las amenazas actuales, ha dinamizado los esfuerzos dirigidos a cubrir la insoslayable necesidad de la cooperación coordinada, tanto en el ámbito nacional como en el internacional.

Ya en el plano organizativo, la aparición del nuevo concepto de la guerra en red (Netwar) ${ }^{8}$ que hoy practica el terrorismo global de Al-Qaeda, ha añadido a los criterios característicos de toda organización funcional -ya asumidos por la mayoría de las agencias- la necesidad de agilizar y flexibilizar sus estructuras, despliegues y redes de intercomunicación interinstitucional y operativas, con el fin de lograr, de una parte, potenciar la interrelación entre los órganos de análisis, aproximar estos a los centros de decisión usuarios de la inteligencia que producen; $y$, de otra, de acortar la distancia existente aún, en tiempo y en espacio, con los órganos de obtención que deben nutrirlos con la información al alcance de sus respectivas capacidades, y a los que se procura, según su naturaleza, más

\footnotetext{
8 El concepto de netwar ha sido desarrollado por los investigadores de la Rand Corporation, John Arquilla y David Ronfeldt. Este tipo de guerra no se refiere a «una guerra en la red" (Internet) (aunque esta constituya el medio ideal para el establecimiento de comunicaciones) sino a la red global de nodos de estructura reticular, que adopta un conjunto de contendientes coaligados en inferioridad en una confrontación asimétrica. Cfr. J. JoRDAN (2002) La seguridad militar en las relaciones de España con los países del Magreb, 2002 (tesis doctoral) U. De Granada, p. 60.
} 
capacidad de acceso a los ambientes propicios a la implantación de potenciales nodos de las redes terroristas, al tiempo que se procura agilizar los sistemas y medios necesarios para la entrega de la información pertinente y en el momento oportuno.

En la actual coyuntura, los principios esenciales que afectan a la gestión de la información y a la elaboración de la inteligencia mantienen su vigencia, así como los fundamentos metodológicos de lo que se conoce como Ciclo de Inteligencia, ya que, éste último, no consiste en otra cosa que en el desarrollo metodológico de la secuencia lógica del pensamiento aplicado a la elaboración de ese tipo peculiar de conocimiento que llamamos inteligencia.

Pero, el trabajo de campo, es decir: en el terreno de la obtención, el desarrollo explosivo de las nuevas tecnologías aplicadas a la información y a las comunicaciones ha multiplicado el número y el alcance de fuentes abiertas que, si bien proporcionan un gran volumen de ella, también exigen sofisticados medios, ajustados procedimientos y esfuerzos muy considerables para seleccionarla, contrastarla, analizarla y evaluarla adecuadamente?

De otro lado, en beneficio de las tradicionales labores de inteligencia en sentido estricto (conocer para proteger y promover los intereses propios), y de Contrainteligencia (conocer para prevenir, detectar y neutralizar agresiones sobre los mismos), las agencias han constatado más que suficientemente, la utilidad de los desarrollos de adquisición técnica y de las herramientas de tratamiento e interpretación de la información especial obtenida a través de ellos; $y$, asi, la transforman, según su procedencia, en inteligencia de señales, de imágenes, acústica, etc., etc., capaces de prestar apoyos - con frecuencia decisivos- a las operaciones de inteligencia en las fases de obtención y aún de elaboración del ciclo de inteligencia; $\mathrm{y}$, al tiempo, han comprobado las ventajas del intercambio y de la colaboración planificada en estos campos, pues son cada vez más conscientes de la necesidad de explotar la complementariedad de sus respectivas capacidades a la hora de cubrir espacios dilatados, lograr mayor alcance, más posibilidades de detección y contraste de la información y de respetar el principio de economía de medios.

9 En la actualidad, navegar por internet proporciona mucha información. Las estimaciones apuntan que la información almacenada se duplica cada dos años. Así, obtener información ya no es un problema, el problema grave es verificar su fiabilidad. Cfr. G. F. TREVERTON, Reshaping Nacional Inteligente in an Age of information, Cambridge, Cambridge University Press, 2001, cap. 1. 
Pero ante los nuevos riesgos, y de forma muy especial, en relación con la amenaza terrorista, la importancia de la información obtenida a través de fuentes humanas, aún habiendo sido siempre predominante en todo lo que se refiere a los círculos de lo secreto y aún de lo discreto, se ha visto multiplicada por la necesidad de acceder a la información solo disponible en el seno de los arriesgados, dispersos y muy restringidos círculos de sus redes y células, difícilmente detectables por cualquier otro medio y aún más difíciles de penetrar.

En resumen: tan amplio panorama de retos y necesidades, impone, en orden a la eficacia y a la economía de medios ya apuntada, una rigurosa aplicación de los principios tradicionales, $y$, con mayor exigencia aún, en todo lo relativo a la determinación y asignación de objetivos, a la construcción de los nuevos marcos de referencia que han de mantenerse actualizados, y a la realización de una labor de planeamiento de la obtención y de la elaboración de inteligencia, ejecutada de forma rigurosa, precisa, flexible, abierta a la complementariedad y a la cooperación entre las distintas agencias, tanto en el plano nacional como en el internacional, y capaz de adaptarse a la volatilidad de las situaciones, a los cambios de escenario y a la aparición de nuevos objetivos.

\section{La Comunidad de Inteligencia (CI): Concepto}

El termino comunidad alude a un conjunto de individuos o entidades reunidos en torno a algo que les es común; es decir a aquello que no siendo privativamente de ninguno ${ }^{10}$, pertenece o se extiende a ellos de una $u$ otra forma. Pues bien, cuando el objeto céntrico de la comunidad resulta ser el logro de lo que llamamos inteligencia, nos hallamos ante esa realidad que se ha dado en llamar Comunidad de Inteligencia. Y al igual que constatábamos que la Inteligencia no sólo era sinónimo de conocimiento y sí, también, acti vidad y organización, se debe afirmar que tal Comunidad -si ha de serlo plenamente- ha de llegar a ser el ámbito natural de cooperación para elaborarla desde las capacidades y responsabilidades de cada uno de sus miembros $\mathrm{y}$, también de acceso a ella en función de sus respectivas necesidades, $\mathrm{y}$, todo esto, merced a una arquitectura orgánica y funcional que lo haga posible; más aún: que lo promueva y exija.

En definitiva, una Comunidad de Inteligencia (CI) consiste en un sistema integrado por las agencias y organismos relevantes en tal materia

10 Cfr. D. de la RAE. 
que sirven a los objetivos estratégicos del Gobierno de la nación, de forma coordinada gracias a la labor de una estructura de superior nivel que promueve y garantiza las relaciones y la conectividad necesaria, a los niveles y por los procedimientos adecuados, y en orden a optimizar los resultados.

En este punto hay que recordar algo que ya se ha anticipado, y que se resume diciendo que nunca, antes de ahora, se percibió la necesidad y la urgencia de articular auténticas y eficaces comunidades de inteligencia capaces de desarrollar esfuerzos complementarios, sinérgicos, interactivos, que cuenten con la compresión de las sociedades a las que sirven y con la cooperación de los diferentes sectores y actores sociales, y orientados al logro de los objetivos e intereses compartidos; porque, como bien dice Robertson ${ }^{11}$, aunque siempre será necesario actuar local y sectorialmente, con frecuencia en beneficio de responsabilidades exclusivas de determinados niveles de responsabilidad y de decisión, hoy se hace inexcusable pensar en términos globales, y promover y perfeccionar medios y procedimientos de trabajo integrado, y sistemas de información e inteligencia compartidas, que hagan posible complementar y coordinar los esfuerzos para alcanzar los objetivos comunes.

El concepto Comunidad de Inteligencia tiene ya historia. Se puede decir que comenzó a acuñarse de modo intencional y funcional, el 22 de enero de 1946, cuando el presidente Truman, fresco aún el recuerdo del fiasco informativo que impidió quizá evitar el desastre de Pearl Harbour, ordenó que todas las actividades que hubieran de desarrollar las diferentes agencias de inteligencia ya existentes relacionadas con la seguridad nacional, y que, hasta entonces, funcionaban sin apenas interconexión, fueran planeadas y desarrolladas, de forma coordinada. Para lo cual encargó, a los secretarios de estado, Guerra y Marina que, con los medios de que disponían cada uno de sus respectivos departamentos, formasen un Grupo de Inteligencia Central puesto bajo la dirección de un Director Central de Inteligencia de designación Presidencial.

Muchos acontecimientos y numerosos cambios se han sucedido desde entonces. Por citar algún ejemplo, baste decir que, hasta ahora, la comunidad de inteligencia de Estados Unidos estaba compuesta, en resumen, por una agencia central (CIA), un amplio abanico de agencias departamentales y otro, no menor, formado por organismos implicados en tareas de inteligencia, todos ellos interrelacionados entre sí a través de una

11 Cfr. R. Robertson, Globalización, 1992. 
compleja red de coordinación interistitucional, a cuya cabeza se encontraba el ya citado Director Central de Inteligencia que lo es también de la CIA.

Pero tras los sucesos del 11 de septiembre, concretamente el 8 de octubre, por orden expresa del presidente Bush, el Gobierno de Estados Unidos inició un largo proceso de reflexión durante el cual, fueron consultadas miles de personas, autoridades y miembros del poder legislativo de los distintos estados de la unión, del Congreso, dirigentes de países extranjeros, expertos del mundo académico y científico, de las Fuerzas Armadas y de Seguridad, de protección civil, del sector privado, etc., en torno a la idea de que la seguridad nacional, y, muy especialmente, la seguridad interior, es una responsabilidad que ha de ser compartida por toda la sociedad en su conjunto; proceso que culminó, el 16 de julio de 2002, tras la firma del Presidente, con la publicación de la Estrategia Nacional de Seguridad elaborada en la propia Casa Blanca.

La finalidad del extenso documento no era otra que la de proporcionar un marco, unos principios y unas políticas dirigidos a la promoción y materialización de la cooperación y convergencia de esfuerzos de todos los actores relevantes en el campo de la seguridad y de la sociedad en su conjunto.

En dicho marco dieron comienzo, en el seno de la CI, profundas revisiones de las estructuras y misiones de las diferentes agencias de inteligencia y de las de los órganos encargados de la coordinación, e iniciativas federales dirigidas, de una parte, a desarrollar una infraestructura básica y segura de intercambio de información, que facilitase un acceso «rápido y total» de los funcionarios responsables de la seguridad interior a la información relevante para sus actividades; y, de otra, "a agilizar el intercambio de información entre la Comunidad de Inteligencia, las fuerzas de seguridad y los órganos gubernamentales de tomas de decisiones», desde la decidida voluntad de impulsar las reformas legales -legislativas y reglamentarias- necesarias para remover aquellas barreras que lo venían dificultando seriamente ${ }^{12}$.

En la misma dirección, también se pusieron en marcha una serie de actuaciones tales como la creación, en noviembre de 2002 , del nuevo Departamento de Seguridad Territorial (con misiones de coordinación en el seno de la CI, para cuestiones de seguridad interna), o la del Centro de

12 Cfr. Estrategia Nacional de Seguridad, Oficina Nacional de Seguridad, 16.07.2002. 
Integración de la Amenaza Terrorista, en enero de 2003; mientras que desde noviembre de 2003, se apuntaba la voluntad de llevar al Gabinete la máxima responsabilidad de la coordinación en el seno de la CI, mediante la creación, en él, de la figura de Director Nacional de Inteligencia en detrimento de las atribuciones que venía ejerciendo el Director de la CIA, en tanto que Director Central de Inteligencia (DCI)

Procesos de revisión análogos se vienen produciendo en la mayoría de los países que sufren idénticas amenazas. Hoy existen, en casi todos ellos, órganos de coordinación de los diferentes servicios de información y de inteligencia. La variedad de sus estructuras es grande; debido, en primera instancia, a la configuración de cada tipo de Estado, al número y naturaleza de los distintos servicios y agencias a coordinar, e incluso a los condicionantes derivados de la escalonada aparición en el tiempo de cada uno de ellos. No debe extrañar, pues, que no resulte fácil establecer homologaciones entre unas y otras, más allá de las funciones genéricas y de los niveles en los que desarrollan su función; pero hay acuerdo general en entender que las cúspides de tales estructuras deben hallarse situadas en los máximos niveles de decisión, dependiendo, en ocasiones, del presidente del Gobierno o del primer ministro, bajo la dirección de un ministro específicamente responsable de la inteligencia o, en otros casos, de órganos colegiados directamente dependientes de la jefatura del gobierno e integrados, de forma variable, por autoridades de alto rango y pertenecientes a los departamentos ministeriales más directamente relacionados con los objetivos marcados a los servicios de inteligencia.

\section{Condiciones de eficacia}

Pero la coordinación no es sencilla, y la labor dirigida al diseño del órgano o sistema que deba realizarla, aún orientada por finalidades bien definidas y por criterios funcionales, no esta libre de tentaciones que, de recaer en ellas, puede conducir a resultados opuestos a los pretendidos. Tentaciones tales como: injerencias en las competencias propias de cada uno de los actores, rigidez y aun pesadez burocrática en los procedimientos de intercomunicación; distanciamiento entre productores y usuarios de inteligencia por la introducción de instancias intermedias que actuarían, fácilmente, como auténticos cuellos de botella que impedirían o ralentizarían el necesario feed back, y que incluso introducirían indeseables interferencias; escasez o proliferación excesiva de órganos específicos 
La Formación de la Comunidad de Inteligencia Española:...

de coordinación de nivel intermedio; disminución de las capacidades de análisis e integración o retardos de la intercomunicación horizontal en tales niveles; deficiente delimitación de los ámbitos competenciales por excesiva compartimentación o por deficiente definición de las finalidades propias de la labor de cada una de las agencias (con la consiguiente confusión acerca del tipo y nivel de la inteligencia a elaborar por cada cual), con el resultado de fomentar la dispersión, las extralimitaciones, el trabajo redundante, las celotipias y aún las pugnas competenciales.

Parece claro, pues, que la coordinación que permita conformar una auténtica y eficaz CI ha de pasar, necesariamente, por el acierto en la elección de una arquitectura en la que estén perfectamente determinados sus miembros, y en la que los órganos y mecanismos de coordinación cumplan una serie de condiciones entre las que cabe destacar: que tengan sus funciones claramente explicitadas en los tres niveles fundamentales de planeamiento, operacional y de difusión; que proyecten su acción sobre todos los espacios definidos por el binomio seguridad-defensa; que promuevan una comunicación rápida y fluida entre los órganos de análisis y las instancias decisorias, y que fomenten -y esto resulta decisivola cooperación entre los miembros de la Comunidad de Inteligencia y la permeabilidad de ésta hacia el resto de la administración, con los sectores políticos, económicos y sociales. Y, todo ello, mediante el desarrollo de redes de cooperación interinstitucional que respondan a criterios de rapidez y eficacia. Es decir, redes compatibles, de alta conectividad, ágiles, flexibles, bien articuladas, adaptables, seguras, que posibiliten reacciones de la Comunidad de Inteligencia adecuadas y en tiempo eficaz.

Pero tales requisitos no son aún suficientes; además se han de cumplir otras condiciones, en mi opinión, también indispensables, tales como: que exista homogeneidad conceptual y terminológica en materia de inteligencia, principios y procedimientos básicos comunes que faciliten el entendimiento y la cooperación, fluido intercambio informativo en el seno de la CI (desde el respeto de los límites de seguridad de las fuentes, procedimientos específicos y operaciones, exigidos por la finalidad que persigue la labor de cada agencia) y, sin menoscabo de la seguridad, aperturas, hacia el resto de la sociedad, capaces de promover en ella una verdadera cultura de inteligencia.

Conviene reparar, muy especialmente, en una de las últimas condiciones que acabo de señalar: aquella que se refiere al intercambio y puesta en común de la materia que las agencias necesitan compartir. Y creo que es necesario hacerlo porque es justamente ahí, en ese ámbito donde aparecen las dificultades más serias para la cooperación entre los servi- 
cios integrantes de la CI, en torno a objetivos que necesariamente han de ser compartidos.

A veces se escuchan opiniones que sostienen que, para solucionar determinados problemas de coordinación e intercambio, sería necesario asignar competencias completas y exclusivas a cada una de las agencias sobre objetivos concretos; $y$, de hecho, son numerosos los casos en que esto es y debe ser así. Pero en relación con los de carácter estratégico no ocurre lo mismo. El ámbito de la seguridad y de la defensa ofrece claros ejemplos de fenómenos que han de ser seguidos por varios servicios de información y de inteligencia de diferentes naturalezas, niveles y finalidades. Ciertamente, cada uno de ellos deberá hacer su trabajo desde la perspectiva que interesa a la finalidad concreta que persigue; finalidades con las que se corresponderán, obviamente, sus misiones y procedimientos respectivos. Pero la realidad de cada fenómeno es indivisible, el objeto de estudio, en todos los casos es uno, y, por tanto, no se puede fragmentar sin desnaturalizarlo. En consecuencia, cada uno de ellos ha de ser contemplado en su unidad para extraer, de él, la información en bruto que permita a cada agencia, tras su análisis y evaluación, proporcionar la noción y los elementos significativos, pertinentes y relevantes necesarios para sus actuaciones en el ámbito específico de su responsabilidad.

En la práctica, proporcionar información procedente de fuentes abiertas o relacionada con las actividades de los individuos, organizaciones y grupos legalmente intervenidas o reguladas por las distintas administraciones, sin menoscabo de los requisitos que las leyes exigen cumplir para proteger el derecho a la intimidad, y al margen de las trabas de carácter burocrático -que de hecho existen en no pocos casos- no suele ofrecer excesivas dificultades.

Otra cosa ocurre cuando se trata de trasladar, intercambiar o compartir información en bruto obtenida por fuentes humanas o mediante herra mientas sistemas y procedimientos técnicos de especial sensibilidad, aún siendo, unas y otros, actuados conforme a ley. En este caso las dificultades aumentan considerablemente. En primer lugar, por razón de la ya señala da sensibilidad de tales fuentes y por la correlativa necesidad de protegerlas. En segundo, porque determinadas informaciones en bruto se encuentran con frecuencia, directamente relacionadas con operaciones en curso que podrían verse comprometidas al ser utilizadas por otras instancias aje nas al curso de los acontecimientos. Y, por último -no se debe ocultar- a consecuencia de reticencias hacia la cooperación derivadas de celotipias, suspicacias y prejuicios (ciertamente indeseables) que existen entre las 
agencias e instituciones de todos los países y, en mayor medida, quizá, en aquellos con escasa tradición en materia de cooperación y coordinación.

$\mathrm{El}$ intercambio entre analistas, es decir, cuando se trata de poner en común el producto inteligencia, las dificultades son menores. Las difusiones suelen circular en horizontal con más facilidad tanto en el ámbito doméstico como en el internacional. Las dificultades aumentan aquí cuando los órganos de análisis de las agencias centrales y las autoridades de coordinación de la CI han de elaborar inteligencia estratégica central integrando informes y documentos procedentes de las agencias y servicios miembros. Pero esto pue de verse paliado o incluso superado -de hecho ya ocurre en los países donde existen- promoviendo, desde tales instancias, grupos de trabajo de análisis y evaluación participados por las agencias afectadas en cada caso.

No obstante lo dicho en relación con las dificultades derivadas del intercambio de información en bruto, la necesidad de actuar globalmente contra amenazas globales de tanta gravedad como el terrorismo transnacional, el crimen organizado de análoga proyección y en sus diferentes vertientes, aconseja la existencia de bases de datos y de inteligencia básica ínteroperables en el seno de la comunidad, que, sin perjuicio de que las agencias o uni dades pueda establecer, en ellas, áreas restringidas por fundadas razones de seguridad de las fuentes u operaciones en curso, permitan el acceso, a las restantes, de los miembros de la CI que pudieran necesitar de ellas.

Más aún: la coordinación en el seno de una CI, en nuestra coyuntura, no puede quedar reducida al mero intercambio de información y de inteligencia. Dicho intercambio, además de nutrir las necesidades de cada servicio, y de facilitar el esfuerzo de los grupos de trabajo que con su participación deban dedicarse al estudio, definición y evaluación de las amenazas, debe también adoptar formulas de cooperación a más bajo nivel y en horizontal: en el plano operacional, manteniendo canales de interlocución directa $\mathrm{y}$, también, mediante equipos, de composición compleja, capaces de canalizar y armonizar la información y la inteligencia necesarias, procedentes de sus respectivos servicios, en apoyo real y eficaz del esfuerzo de quienes han de asumir la responsabilidad directa de neutralizar cada una de ellas a nivel táctico.

\section{La formación de la Comunidad de Inteligencia en España}

En España, con la promulgación de la Ley 11/2002, de 6 de mayo, reguladora del CNI se ha optado por un sistema nacional de inteligencia que se basa en la articulación de una Comunidad de Inteligencia com- 
puesta por «todos los servicios de información e inteligencia del Estado»"13 y por un órgano del máximo nivel gubernamental: la Comisión Delegada del Gobierno para Asuntos de Inteligencia (CDGAI), encargada de «velar por la coordinación del Centro Nacional de Inteligencia (CNI), de los servicios de información de los Cuerpos y Fuerzas de Seguridad del Estado y los órganos de la administración civil y militar» ${ }^{14}$.

La misma Ley establece que, bajo la presidencia de un Vicepresidente del Gobierno, la Comisión esté integrada por los ministros de Asuntos Exteriores, Defensa, Interior, y Economía, así como por el Secretario General de la Presidencia, el Secretario de Estado de Seguridad y el Secretario de Estado Director del Centro Nacional de Inteligencia, que actuará como Secretario ${ }^{15}$, y al que le corresponde también desempeñar las funciones de Autoridad Nacional de Inteligencia y Contrainteligencia ${ }^{16}$ y las de Director del Centro Criptológico Nacional ${ }^{17}$; y determina como funciones a desarrollar por tan alto órgano las siguientes:

a) Proponer al Presidente del Gobierno los objetivos anuales del Centro Nacional de Inteligencia que han de integrar la Directiva de Inteligencia.

b) Realizar el seguimiento y evaluación del desarrollo de los objetivos del Centro Nacional de Inteligencia.

c) por la coordinación del Centro Nacional de Inteligencia, de los servicios de información de los Cuerpos y Fuerzas de Seguridad del Estado y de los órganos de la Administración civil y militar.

A la luz, pues, de lo legislado, se aprecia la voluntad política de iniciar un proceso dirigido a la formación en España de una comunidad de

\footnotetext{
13 Artículo 6.1 de la Ley 11/2002, de 6 de mayo, reguladora del Centro Nacional de Inteligencia.

14 Ibídem. Artículo 6, ap. 4, c).

15 Ibídem. Artículo 6, ap. 4, c).

16 Esta figura, existente en diferentes países de nuestro entorno, juega un importante papel en la coordinación en el seno de las comunidades de inteligencia. Su antecedente cabe situarlo en el memorando que el Presidente Truman dirigió a los secretarios de Estado, de Guerra y de Marina, el 22 de enero de 1946 -que hemos tenido ocasión de citar-y que inició el proceso de creación de una agencia de inteligencia central (la CIA), bajo la dirección del Consejo de Seguridad Nacional, y cuyo Director desempeñaría las funciones de Director de Inteligencia Central. Este proceso culmino con la promulgación de la Ley de Seguridad Nacional de 1947.

${ }_{17}$ Artículo 9, ap. 2, f) de la Ley 11/2002, de 6 de mayo, reguladora del Centro Nacional de Inteligencia.
} 
La Formación de la Comunidad de Inteligencia Española:...

inteligencia en sentido estricto, y, por tanto, ceñida a su concepto y finalidad. Un sistema, pues -recordémoslo- formado por las agencias y organismos relevantes en tal materia, que sirven a los objetivos estratégicos del Gobierno de la nación, de forma coordinada, gracias a la labor de una estructura de superior nivel que promueve y garantiza las relaciones, la conectividad y las sinergias necesarias, a los niveles y por los procedimientos adecuados, en orden a optimizar los resultados y favorecer la economía de medios.

Y véase cuales son las instancias que parece contemplar el legislador en relación con sus integrantes: en primer término, como núcleo central de la Comunidad, una única agencia de inteligencia de nivel nacional, el CNI, los Servicios de Información de los Cuerpos y Fuerzas de Seguridad del Estado, en el ámbito del Ministerio del Interior, y el -en proceso de formación y consolidación- Centro de Inteligencia de las Fuerzas Armadas (CIFAS) que, a las órdenes del Jefe del Estado Mayor de la Defensa (JEMAD), debe atender a las necesidades de inteligencia de las Fuerzas Armadas. Y, en un segundo plano, todos aquellos organismos de la Administración, tanto civiles como militares, cuyas funciones y ámbitos de trabajo resultan especialmente relevantes en materia de información y de inteligencia. En este último punto, parece del todo lógico pensar también en todos aquellos organismos que desarrollan tareas de tal índole, en ministerios tales como Hacienda o Exteriores, cuyos titulares, por otra parte, son miembros de la Comisión Delegada del Gobierno para Asuntos de Inteligencia.

Pero si miramos un poco más allá de la letra, y se reflexiona sobre los conceptos de Comunidad de Inteligencia y de Administración en sus sentidos amplios, habrá que reparar en el papel que deben jugar, en relación con la Comunidad, los organismos e instituciones dependientes de la Administración central, de las administraciones autonómicas e incluso de las locales relacionadas con la seguridad.

$\mathrm{Y}$, por último, hay que tener muy en cuenta que la labor de los servicios de información y de inteligencia, en el mundo actual, en nuestras sociedades de la información, no se reduce al ámbito de lo secreto, ni se dedica a bucear en un mundo de sombras, de espaldas y a cubierto de la sociedad de la que forman parte y a la que deben servir; y sí se aplica, por el contrario, con especial énfasis, a la prevención de los riesgos y de las amenazas que se ciernen sobre ella, contando con ella, con su apoyo; con el de apoyo, muchas veces, de organizaciones y expertos ajenos a los gobiernos: del mundo académico, de la economía y de la empresa, de los think tanks... En definitiva, con el apoyo de esa sociedad viva, estrecha- 
mente vinculada a la realidad, que, de permanecer abiertos a ella, evita sufrir uno de los males más peligrosos para un servicio de inteligencia: el ensimismamiento, la desconexión con el referente contextual que aísla y conduce al pensamiento circular y acrítico.

El proceso de formación de la Comunidad de Inteligencia española, en mi opinión, no ha concluido. Todavía ha de recorrer un buen trecho hacia su debida configuración funcional. Queda por delante completar su arquitectura resolviendo, entre otras, cuestiones en absoluto baladíes: definir, desarrollar e implementar los medios y procedimientos que permitan a la CDGAI el seguimiento y potenciación de la coordinación entre los integrantes de la Comunidad de Inteligencia y, la de estos, con los restantes organismos del Estado con capacidades informativas y para la elaboración de inteligencia; establecer, de forma precisa, las funciones de la Autoridad Nacional de Inteligencia y Contrainteligencia y su relación funcional, en materia de coordinación, con la CDGAI, y en la cual, su titular, el SE Director del CNI actúa como Secretario; y promover, tutelados y orientados por la Comisión y en beneficio de la Comunidad, grupos de trabajo, centros de análisis interdepartamentales que atiendan, de forma continua, al estudio factorial de los fenómenos que constituyen objetivos estratégicos que, por su complejidad, requieren ser abordados de forma multidisciplinar, y que, con frecuencia, trascienden el ámbito doméstico y exigen la cooperación internacional.

Pasos importantes ya se han dado en este difícil campo de la coordinación: existe, ya, y operante, un órgano del máximo nivel, la CDGAI, que, a través de la Directiva de Inteligencia-que somete, anualmente, a la aprobación del Presidente del Gobierno, en Consejo de Ministros- define, con claridad y precisión, los objetivos del CNI para cada ejercicio, y establece los principios generales de coordinación con los servicios departamentales competentes en el ámbito de algunos de sus objetivos. Se ha avanzado notablemente en el proceso de desarrollo y consolidación del Centro de Inteligencia de las Fuerzas Armadas (CIFAS), en la dirección marcada por la Directiva de Defensa Nacional vigente y en orden a su coordinación con el CNI y con los Cuerpos y Fuerzas de Seguridad del Estado. Y, en el Ministerio del Interior, para la coordinación informativa en relación con el terrorismo, por Acuerdo de Ministros de fecha 28 de mayo de 2004, se creó el Centro Nacional de Coordinación Antiterrorista (CNCA) que, a nivel departamental, analiza y evalúa la amenaza terrorista, que está integrado por personal especializado del Cuerpo Nacional de Policía y de la Guardia Civil, adscritos a la Secretaría de Estado de 
Seguridad, y por una Unidad de Apoyo del CNI con quien se mantiene vinculada funcional, orgánica y disciplinariamente.

En el campo de la Inteligencia de Señales, la cooperación de los organismos especializados del CNI con sus homólogos de las Fuerzas Armadas tiene ya una larga tradición de asiduidad y eficacia, que se verá seguramente reforzada y ampliada tras la conclusión del proceso de organización y asentamiento del Centro de Inteligencia de las Fuerzas Armadas. Y, por lo que respecta a la seguridad de los sistemas y procedimientos de transmisión y almacenamiento de la información clasificada, el Centro Criptológico Nacional (CCN), creado por la Ley 11/2002 de 6 de mayo, Reguladora del Centro Nacional de Inteligencia, bajo la dirección de su Secretario de Estado Director, asume, al servicio de la CI y del resto de las administraciones civiles y militares del Estado, la responsabilidad de desarrollar las funciones y actividades necesarias para proporcionarla.

Por último, creo que se debe poner de relieve que se han ido intensificando y llenando de contenido las relaciones bilaterales tradicionales, abriéndose o reforzándose otras y, en no pocas ocasiones, desarrollando proyectos y actividades conjuntos y en colaboración con el mundo acadé mico, de la investigación -como es el presente caso- de la empresa y de la comunicación, de los que son también esperables resultados positivos de cara a un mejor conocimiento, por parte de la sociedad, de la necesidad, alcance y contenido de la función de los servicios de información y de inteligencia, y de la importancia que para ellos tiene su apoyo para mejor servirla ${ }^{18}$.

El proceso, pues, ha logrado serios avances. El impulso continúa; y los éxitos alcanzados en la lucha contra el terrorismo islamista, tras el 11 de marzo, y frente al de ETA, no son ajenos a tales logros. Pero, como ya se anticipó, la CI española debe seguir desarrollando su arquitectura y sus mecanismos de intercambio y cooperación en consonancia con nuestro sistema de inteligencia, aprovechando las experiencias ajenas que se hayan demostrado eficaces, y procurando eludir los errores -no nos faltarán ejemplos de ellos- que se cometieron -y aún se cometen- en otros países de nuestro entorno.

18 En este orden de ideas es interesante, recordar la opinión de Sherman Kent al respecto: «En cierto sentido, las organizaciones de inteligencia deben parecerse un poco a una gran universidad ..." S. Kent, Inteligencia Estratégica, Buenos Aires, 1994, p. 90. 